\title{
Correction to: On Structured Surfaces with Defects: Geometry, Strain Incompatibility, Stress Field, and Natural Shapes
}

\section{Ayan Roychowdhury ${ }^{1}$ Anurag Gupta ${ }^{1}$}

Accepted: 5 July 2021 / Published online: 23 November 2021

(c) Springer Nature B.V. 2021

\section{Correction to: Journal of Elasticity (2018) 131: 239-276 https://doi.org/10.1007/s10659-017-9654-1}

1. In the right hand sides of Eqs. (39), (55)-(58), (59)-(62), (74)-(76), and (78)-(80), replace the partial derivative operator with $\tilde{\partial}$.

2. Consequent to Item 1, Eqs. (68)-(70) should be replaced by

$$
\begin{aligned}
K_{1212}+\left[b_{11} b_{22}-b_{12}^{2}\right] & =4 \partial_{[1}\left(\sqrt{a} a_{2] \sigma} J^{\sigma}\right)-2 C_{[1|\mu 2|} C_{2] 1}{ }^{\mu}-a\left(J^{3}\right)^{2}, \\
-\partial_{2} b_{11}+\partial_{1} b_{12} & =\partial_{1}\left(\sqrt{a} J^{3}\right)+2 a J^{2} J^{3}+2 \sqrt{a} J^{\sigma}\left(b_{1}^{2} a_{\sigma 2}-b_{2}^{2} a_{\sigma 1}\right), \\
-\partial_{2} b_{21}+\partial_{1} b_{22} & =\partial_{2}\left(\sqrt{a} J^{3}\right)-2 a J^{1} J^{3}++2 \sqrt{a} J^{\sigma}\left(b_{2}^{1} a_{\sigma 1}-b_{1}^{1} a_{\sigma 2}\right) .
\end{aligned}
$$

Henceforth, Eqs. (82)-(83) and (84), should be

$$
\begin{aligned}
\Lambda_{11}^{p} \Lambda_{22}^{p}-\left(\Lambda_{(12)}^{p}\right)^{2} & =2\left(J_{, 1}^{2}-J_{, 2}^{1}\right)-\left(J^{3}\right)^{2}, \\
\Lambda_{11,2}^{p}-\Lambda_{(12), 1}^{p} & =J_{, 1}^{3}+2 J^{2} J^{3}+2 J^{1} \Lambda_{22}^{p}-2 J^{2} \Lambda_{(12)}^{p}, \\
\Lambda_{(12), 2}^{p}-\Lambda_{22,1}^{p} & =J_{, 2}^{3}-2 J^{1} J^{3}+2 J^{2} \Lambda_{11}^{p}-2 J^{1} \Lambda_{(12)}^{p} .
\end{aligned}
$$

These three relations have earlier appeared as Eq. 35.82 in Derezin, Shell-like Structures, Vol. 15, pp. 531-547. Springer, 2011.

3. In Eqs. (74) and (91), the term $2 \sqrt{A} A_{\sigma[1} \bar{\partial}_{2]} J^{\sigma}$ should be replaced by $-4 \sqrt{A} A_{\sigma[1} \tilde{\partial}_{2]} J^{\sigma}$.

Publisher's Note Springer Nature remains neutral with regard to jurisdictional claims in published maps and institutional affiliations.

The original article can be found online at https://doi.org/10.1007/s10659-017-9654-1

A. Gupta

ag@iitk.ac.in

1 Department of Mechanical Engineering, Indian Institute of Technology Kanpur Kanpur, 208016, India 